\title{
La Ducasse d'Ath au rythme des (r)évolutions, 1786-1819
}

\author{
Adrien Dupont
}

Adrien Dupont : Adrien Dupont est licencié en histoire de l’Université catholique de Louvain, archiviste de la Ville d'Ath, secrétaire du Cercle royal d'histoire et d'archéologie d'Ath et membre du Conseil d'ethnologie de la Fédération Wallonie-Bruxelles.

DOI: $10.25518 / 1370-2262.254$

\section{Résumé :}

La période de 1786 à 1819 est probablement l'époque où il a été le plus légiféré sur la ducasse d'Ath. Les conceptions religieuses de Joseph II, l'acharnement des patriotes jacobins, la politique thermidorienne, la restauration napoléonienne du culte et l'influence de l'administration calviniste des Pays-Bas ont asséné des coups de boutoir à la fête séculaire. D'une fête d'origine et à caractère religieux, la Ducasse se mue en une fête communale. Par-delà l'arsenal normatif, les habitants d'Ath ont su conserver et maintenir intact l'élément culturel identitaire de leur cité. La transmission de leur patrimoine immatériel a ainsi surmonté les guerres et les révolutions.

Mots-clés : Ath, Ducasse, Joseph II, Procession

\section{Abstract :}

The period from 1786 till 1819 is probably the period when it was the most legislated on the Ducasse of Ath. The religious conceptions of Joseph II, the doggedness of the patriots Jacobins, the thermidorienne policy, the napoleonic restoration of the cult and the influence of the Calvinist administration of the Netherlands hurled thrusts at the secular holiday. Of a holiday of origin and with religious character, Ducasse moved in a communal fair. Beyond the normative arsenal, the inhabitants of Ath knew how to preserve and maintain intact the identity cultural element of their city. The transmission of their immaterial heritage so overcame the wars and the revolutions.

\section{Introduction}

Le 25 novembre 2005, les instances de l'Unesco élevaient au titre de chef-d'œuvre du patrimoine oral et immatériel de l'humanité1 les "Géants et dragons processionnels de Belgique et de France " 2 , au sein desquels la Ducasse d'Ath occupe une place de choix. Parmi les critères de définition du patrimoine culturel immatériel, il faut entre autres retenir le critère de transmission de génération en génération d'une pratique culturelle. Le propos de cet article s'attache dès lors à examiner comment la transmission de la tradition athoise s'est opérée durant les temps troublés de la charnière entre les XVIII ${ }^{\mathrm{e}}$ et $\mathrm{XIX}^{\mathrm{e}}$ siècles.

\section{Une Ducasse aux lointaines origines : un état des lieux à la fin du XVIIIe siècle}

Située au confluent des deux Dendre, la ville neuve d'Ath est créée par le comte de Hainaut, 
Baudouin IV, au pied de son château, la Tour Burbant, vers 1166. La cité devient un centre économique, administratif et religieux durant le $\mathrm{XIV}^{\mathrm{e}}$ siècle. C'est au tournant du $\mathrm{XV}^{\mathrm{e}}$ siècle qu'est attestée la procession de la dédicace de l'église à saint Julien de Brioude, fêté le 28 août $\underline{3}$. La procession va progressivement s'enrichir d'une série de représentations de l'Ancien et du Nouveau Testament ainsi que de la Légende dorée. Le premier géant connu est le Cheval Bayard, réalisé en 1462, pour révérender ladite procession et la boine ville. Mentionné pour la première fois en 1481, Goliath combat le berger David comme dans la Bible. Le Conseil de ville le dotera d'une épouse en 1715. Le héros Samson est cité en 1679 comme une réalisation des canonniers bourgeois 4 .

Au fil des siècles, la Ducasse d'Ath n'a cessé d'évoluer. Régulièrement, des événements militaires ont eu un impact direct sur le cours de son déroulementㅁ․ En 1750, l'historien Gilles-Joseph de Boussu souligne déjà que les guerres ont souvent suspendu l'ostentation de ces fêtes ravissantes $\underline{6}$. S'ensuit la période troublée des révolutions, considérée dans cet article. Ici, la Ducasse est contrainte de se plier, non plus à la force des armes, mais à la force des lois.

Après la Contre-Réforme, le cortège de la dédicace perd nombre de ses histoires religieuses et tend à devenir plus festif. Les spectacles différens qui l'accompagnent sont si agréables et si diversifiés, que l'on peut dire qu'elle - la procession - est aussi charmante que pieuse, assure Gilles de Boussu en 1750. Lorsqu'en 1781, Joseph II accède à la souveraineté des territoires des Habsbourg d'Autriche, la kermesse d'Ath est un savant enchevêtrement d'activités : procession des confréries, des gildes et de leurs figures gigantesques, scènes bibliques dont le combat de David et Goliath, vêpres du samedi auxquelles participe le couple gigantesque, salves des canonniers bourgeois, cérémonies rehaussées de musiciens, etcz.

En 1966, René Meurant étudie un programme montrant la composition de la procession athoise à la fin du XVIII ${ }^{\mathrm{e}}$ siècle $\underline{8}$. Cet ordonnancement est proche de la description de la dédicace athoise publiée par l'écrivain tournaisien, Adrien Hoverlant de Bauwelaere en 1820․ La première partie du défilé s'ouvre par le groupe des orphelins et des élèves des petites écoles. Les confréries professionnelles occupent la plus grande partie du cortège. Elles sont suivies des serments militaires. Les étudiants du Collège inaugurent la seconde partie de la procession. Le géant Goliath et sa femme annoncent les autorités civiles siégeant sur le char de la ville. Les statues de la Vierge portées par les Capucins et les Récollets précèdent le clergé des deux paroisses de Saint-Julien et de Saint-Martin. La marche se clôture avec le gouverneur de la ville et le châtelain. Parmi cette trentaine de groupes, les confréries étalent leurs vitalités. Les orfèvres et les commerçants du textile présentent chacun un char de triomphe. Les bouchers promènent saint Jean-Baptiste et son agneau. D'autres sortent leurs géants d'osier : les boulangers un cygne, les tailleurs un aigle, les poissonniers un triton, les tireurs à l'arc un tyran, les canonniers un Samson. Mr et Mme Goliath, accompagnés du berger David, défilent avec les autorités civiles.

\section{6 : une réforme de Joseph II}

Mais dès son avènement, le despote éclairé promulgue des décrets touchant l'organisation de l'État et les pratiques religieuses. Ainsi, le 11 février 1786, il s'en prend aux kermesses ou dédicaces $\underline{10}$. Le projet du décret définit une kermesse ou dédicace comme une fête annuelle qui se fait dans chaque paroisse tant des villes que du plat pays en mémoire de la consécration de l'église paroissiale ... Cet anniversaire se trouve établi dans le fait par un usage constant et universel depuis plus de dix siècles mais tombe à des jours différents selon la paroisse11. L'empereur veut donc remédier 
aux inconvéniens multipliés qu'entrainent, au préjudice de la Religion et de l'Etat, ces fêtes au caractère hétérogène. Désormais, toutes les kermesses se tiendront le même jour, partout sur l'étendue des Pays-Bas autrichiens, à savoir le second dimanche après Pâques. Le Grand Bailli de Hainaut ordonne la diffusion du décret le 20 février et il est affiché à Ath le 24 du même mois 12 .

En séance du 4 avril 1786, la Chambre échevinale d'Ath, conformément au prescrit impérial, ordonne que la procession générale de cette ville se fera le 30 avril de cette année premier dimanche d'après le Quasimodo (...) comme elle se faisoit ci-devant le quatrième dimanche d'août1․ Dans l'esprit des édiles, cette mesure comprend également la cérémonie qui etoit d'assister aux vespres, y ayant toujours été invités de temps immemorial par le curé de la paroisse de St Julien qui venoit avec son clergé prendre le Magistrat à l'hotel de ville14. Le clergé, dirigé par le doyen PhilippeJacques Defrenne15, décide de ne pas se prêter à cette modification arguant que le décret n'était pas d'application dans le diocèse de Cambrai16.

Le 15 avril, le Magistrat athois interroge le Conseil Privé à Bruxelles sur le point que le clergé soit en droit de refuser de se conformer à la nouvelle législation $\underline{17}$. La réponse de Bruxelles arrive le 26 avril18. Le souverain y réaffirme la suppression de la procession du $4^{\mathrm{e}}$ dimanche d'août, son déplacement au 30 avril, son déroulement selon le rite du jour de la dédicace et l'autorisation au Magistrat d'y assister en corps19. Il interdit expressément que cette procession soit accompagnée d'aucun objet profane qui puisse distraire le peuple de la dévotion avec laquelle il doit assister à cette cérémonie religieuse.

Peu de temps après, le 10 mai 1786, l'empereur promulgue un second édit concernant les processions et jubilés 20 . À l'exception des rogations, il n'est plus autorisé, dans chaque paroisse, que deux processions : l'une le jour de la Fête-Dieu et l'autre un jour à désigner par l'ordinaire en chaque diocèse. L'archevêque choisit le 15 août, jour de l'Assomption de la Vierge, pour l'étendue de son archevêché de Cambrai21. L’article deux de l'édit prévoit qu'il ne sera plus permis de porter de statues ni d'images quelconques, non plus des enseignes de métiers, vêtements extraordinaires ou autre bigarrures semblables dans les processions ni les faire accompagner d'aucune musique. Cet article impactera le cours des choses à Ath sous deux aspects.

Quoi qu'ait pu écrire l'abbé Plumet, les choses ne sont pas passées sereinement entre le Magistrat et le doyen Defrenne avant les troubles révolutionnaires en provenance de France22. Concomitamment à la législation sur les processions, le souverain proclame la suppression des confréries le 8 avril 1786. Acceptant cette suppression, l'abbé Defrenne n'en tergiverse pas moins à propos de la décharge des fondations des associations supprimées et s'attire les foudres des échevins $\underline{23}$, au point que ceux-ci recourent à l'intervention de Bruxelles 24 . Le Conseil Privé requiert alors un rapport de l'officier fiscal du Hainaut, M. Papin, qui sera remis le 30 août 25.

Le 16 avril 1786, un courrier du doyen prouve que le prêtre opine dans le sens du décret portant sur les processions. Selon lui, aux clergés des paroisses de la ville et à tous les gens sensés du peuple, les extravagances de ces processions déplaisent depuis longtemps et (qui) sont fort couteuses à la ville. Le cortège du 30 avril serait alors pour lui l'occasion de retirer une quantité d'objets profanes et extravagants qui pourrait distraire le peuple de la dévotion et d'abolir en outre toutes les cérémonies et démonstrations qui étoient d'usage la veille de la kermesse. Plus tard, le Magistrat ne manquera pas de contre attaquer lorsqu'à la Fête-Dieu du 15 juin, le doyen aura sorti quatre étendards de confréries, dits gonfalons, peints de différentes figures. Le Fiscal Papin n'en blâmera pas le curé, d'autant qu'il constate qu'un grand nombre de personnes de tout état et 
condition pensent qu'il est indifférent ... que l'on fasse ces sortes de processions dans l'intérieur ou autour des murs des églises surtout lorsqu'il n'y a qu'une nefve dans l'église ou quand il y a affluence de peuple.

Par ailleurs, le décret de mai 1786 et son projet26 interdisent aux corps de métiers et aux administrations des villes de pratiquer ces sortes de jubilé27 par des fêtes, ornemens, des batimens, cavalcades ou autrement. Le 15 mai, considérant probablement le concours d'étrangers très considérable, qui y arrive de toute part souligné par G. de Boussu en 1750 et pour éviter ce qui se passe au Saint Sang à Bruges28, M. de Reul, du Conseil Privé, somme la ville d'Ath de vendre toutes les décorations et autres objets que vous pourriez avoir conservé jusqu'ici pour la célébration de quelque jubilé29.

Par délibération du 6 juin 1786, les échevins décident de faire vendre par recours public tous les vieux bois, ferailles et autres ustencils appartenant tant à la ville qu'à la mambournie de Saint Julien30. La comptabilité communale atteste bien une vente le 27 juin suivant pour des boiseries de la ville entreposées au château et des bois de la chapelle Notre-Dame du Rosaire $\underline{31}$. Pour la mambournie de l'église, le cas n'est pas neuf. Régulièrement, le chapitre 14 de ses recettes compte un revenu provenant de la vente de vieux bois à des particuliers sur ordre du Magistrat $\underline{22}$. Seul le compte du massard communal clôturé en octobre $1786 \underline{33}$ signale que J.-P. Routier $\underline{34}$ aurait conduit les figures gigantesques et le char de la ville sur la grand place pour y être vendus. En l'état actuel des dépouillements, rien ne permet cependant d'avancer que la vente ait bien eu lieu $\underline{35}$.

Ainsi, dès août 1786, les processions publiques dans les rues de la ville se limitent à la fête du Corps du Christ et aux rogations comme l'attestent les semainiers de l'église Saint-Julien36. Dans une mercuriale datée du 28 mai 1787 et distribuée à Ath37, les Montois, relèvent le sentiment de tristesse qui peut tirailler les Athois de ne plus voir leur défilé : Seriez-vous insensibles aux larmes de Goliat qui gémit de ne plus être la vénération du peuple athois dans votre procession ?38 Partie de cette mercuriale pourrait ne constituer qu'une réplique au courrier du doyen Defrenne du 16 avril 1786 adressé aux instances de Bruxelles, si tant est que les Montois eussent eu connaissance de cette missive. Le curé y cite une lettre en date du 9 avril 1786 qu'il a reçue de l'avocat Raout, un Athois résidant à Mons 39. Pour étayer sa démonstration, il donne l'exemple de Mons où la procession est redevenue toute spirituelle : le dragon, les chinchins et la cérémonie du lumeçon, de même que les tirs des canonniers, ont été supprimés.

\section{0 : un retour de courte durée}

Un vent de révolte va désormais souffler sur la politique de Joseph II. Dans un contexte d'opposition aux réformes du roi sacristain, Ath n'est pas épargnée느. Dès 1787, des tensions apparaissent entre notables athois, royalistes ou patriotes; des émeutes sont latentes en juin et à la Toussaint. Dès que la rupture est consommée entre les États et l'Empereur, un nouveau climat insurrectionnel règne dans la cité de Goliath en octobre 1789. En décembre, un comité de patriotes se met en place. Les États de Hainaut viennent à la rescousse de la ducasse d'Ath. Le 12 août 1790, ils annulent les effets des édits du monarque éclairé41, en ce compris les édits de 1786 relatifs aux kermesses.

Aussitôt dit, aussitôt fait, les figures gigantesques athoises sont restaurées et sortiront comme à l'accoutumée le $4^{\mathrm{e}}$ dimanche d'août 1790 . Perruquier, peintre, mandelier, tailleur, modiste, tous $\underline{42}$ sont mis à contribution pour raccommoder les géants et les chevaux jupons 43 . Il en coûtera 203 livres 10 sous 3 deniers à la Ville ... qui ne remboursera l'avance de cette somme au receveur que 
le 14 février $1792 \underline{44}$.

Concernant les deux géants, Goliath et sa femme, les mentions comptables sont explicites. Il s'agit bien d'une restauration et de raccommodage. Le perruquier Noyon est payé « pour restauration de la chevelure », le tailleur Michel pour « raccommoder les dites figures », le mandelier Dubois « pour avoir raccommodé les panniers ». Ce Jean François Joseph Dubois a surtout aussi été rémunéré «pour avoir remonté les figures ». Il est dès lors on ne peut plus clair que les géants n'ont pas été vendus en juin 1786. Ils ont probablement été démontés et remisés dans une quelconque grange de la ville ! Comme Christian Cannuyer l'a avancé, le défaut d'acquéreur à la vente du matériel peut aisément être perçu comme une manière pour la population de marquer sa désapprobation envers les velléités du régime en place à Bruxelles $\underline{45}$.

Le cortège sortira les années suivantes; divers travaux sont effectués au matérielú6. En 1791, le mandelier Dubois réalise deux paniers neufs et le ferblantier Walem répare les boucles d'oreille de Madame. En 1792, Marie Dereux fournit des éléments d'ornementation. En 1793, un panier est ajusté et les perruques sont nettoyées. A priori, selon ces mentions, la Ducasse ne semble pas trop souffrir des aléas politiques et militaires des premières années de la décennie $1790 \underline{47}$.

L'ordre autrichien est rétabli au printemps 1791. Les forces libératrices françaises obtiennent la victoire de Jemappes le 6 novembre 1792. Elles pénètrent dans Ath le 9 novembre. Une société des Amis de la Liberté et de l'Egalité se crée sur le modèle jacobin en ville sous le nom de Société populaire. Cependant, le 18 mars 1793, les Autrichiens défont les révolutionnaires à Neerwinden. Le Magistrat ancien est rétabli dans ses fonctions. Une chasse aux sorcières a lieu. Des actes de vengeance sont perpétrés contre les symboles de la République : le local de la Société populaire est saccagé et son mobilier est brûlé.

Valenciennes et Condé sont prises les 8 et 10 juillet 1793. Afin de commémorer l'événement, le maire d'Ath Nicolas Delescluse ordonne des réjouissances publiques et fait faire en osier une figure qu'il fait appeler Victoire $\underline{48}$ pour perpétuer la mémoire de la prise des villes par les impériaux... Elle paradait à la cermesse d'Ath et dans les processions à côté d'autres figures que les peuples de ce pays étoient dans l'habitude de faire promener à des époques périodiques $\underline{4}$.

\section{4 : l’autodafé révolutionnaire}

Cette allégresse royaliste n'aura qu'un temps. La victoire du général Jourdan à Fleurus le 26 juin 1794 marque le retour du régime révolutionnaire dans nos régions. Le 7 juillet 1794 (9 messidor an II), un détachement de cavalerie prend possession d'Ath et les édiles communaux athois invitent les habitants à illuminer leurs façades à 9 heures du soir en signe de réjouissance pour l'entrée

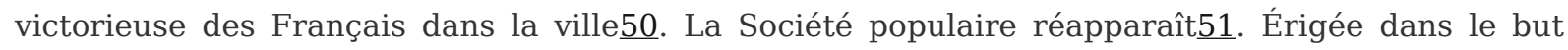
de surveiller les ennemis du peuple, ses actions sont teintées d'accents déchristianisateurs et anticléricaux. Ses membres vouent une haine féroce aux ennemis de la République et s'emploient à faire tomber les signes distinctifs de l'Ancien Régime.

Ainsi, la Société populaire adresse un courrier à la municipalité, réclamant la tête des anciennes figures de la ducasse d'Ath et leur destruction par le feu. Le conseil de la commune statue sur ce point le 5 fructidor an II (22 août 1794) et laisse à la Société le choix du mode de l'anéantissement de ces figures et lui octroie plein pouvoir à cet égard $\underline{52}$.

Le 6 fructidor, le commissaire civil Jasmin Lamotze écrit au magistrat : Le tems est passé, citoyens, 
ou les arlequinades et les momeries religieuses etoient les principaux reports de la ressource des prêtres et des aristocrates. La procession qui se faisoit ci-devant le jour de votre dedicasse etant encore un de ces signes honteux pour les vrais republicains, je vous invite au nom du bien public et au besoin vous fait defense, citoyens, de faire faire cette procession parmi votre commune ainsi qu'elle se faisoit dans l'Ancien Regime " $\underline{53}$. Le même jour, le Conseil de ville communique l'interdiction de danses et parties de plaisirs ordonnée par le commandant de la place pour le 7 fructidor, $4^{\mathrm{e}}$ dimanche du mois d'août $\underline{54}$.

Le 8 fructidor, le maire et les officiers municipaux communiquent la décision du 5 fructidor aux membres de la Société populaire, en ces termes : Il y a longtems, citoyens, que toutes personnes sensées ont senti le rédicule de ces figures gigantesques et autres momeries, qui aux yeux de certains, on pourroit dire de la multitude, paroissoient etre un spectacle bien beau et tel, selon eux, qu'il faisoit le brillant de la procession de la dedicace de cette ville. L'enfantement de pareilles bouffonnerie, il faut en convenir, ne peut etre du qu'au siecle d'ignorance qui la vu naitre et il n'appertenoit qu'a celui où la raison repend a présent son empire, de faire disparoitre toutes ces absurdités, en eclairant les peuples $\underline{5}$. Ils commencent donc par minimiser le caractère sentimental qui unit la ville à ses géants en les reléguant à un rôle accessoire qui ajoute du panache à la procession, un peu comme de Boussu l'écrivait déjà en 1750. Le temps présent recommanderait d'exclure ces absurdités de la fête ; l'abbé Defrenne le préconisait aussi quelques années auparavant mais pour en revenir à une spiritualité plus authentique.

Ensuite, le maire et les municipaux vont tenter de leur faire oublier l'idée de brûler les géants. Après avoir cité les écrits du commissaire Lamotze, ils font remarquer qu'il n'évoque pas le feu mais se borne à interdire la procession ainsi qu'elle se faisoit ci-devant. Bien que l'anéantissement ait été prononcé en conseil, le choix du procédé est néanmoins laissé à la Société populaire.

Les membres de cette société n'en démordront pas et, comme en souvenir de la destruction de leur local par le feu, ils procèdent à l'autodafé $\underline{56}$. En apostille à la délibération du 5 fructidor, il est déclaré qu'il s'est déroulé le 11 fructidor, soit le 28 août, jour de la dédicace de Saint-Julien. Le 13 fructidor, le receveur communal verse 26 livres 15 sous à la citoyenne Dereux $\underline{77}$ pour salaire et livrance faite pour habiller Goliath et sa femme à l'effet de les conduire à l'autodafés.

Le contexte dans lequel cet événement se déroule est classique en temps de troubles: Goliath et Samson ont connu des éclipses durant les guerres모. Le cas de 1786 en est encore une belle illustration. Cependant, ici, en 1794, les membres de la Société populaire veulent véritablement éradiquer les momeries d'un autre âge et ils ont recours au feu. Les figures gigantesques sont présentées comme un signe de l'Ancien Régime, un symbole d'un état d'esprit qu'il faut faire disparaître. La destruction d'objets porteurs de sens est désormais intentionnelle, volontariste et non plus un oubli dans quelque placard en attendant des jours meilleurs.

Il faut relever que les textes ne parlent que des géants de la ville, appartenant au patrimoine communal défini à la suite du règlement financier de 1769. D'après la lettre du 8 fructidor, toutes ces figures doivent être données pour en être disposé à votre volonté. Ainsi, Goliath, sa femme et Mademoiselle Victoire $\underline{60}$ seront la proie des flammes. Les autres géants (comme l'Aigle, le Cygne, le Triton ou Samson) appartiennent à des confréries; rien ne prouve qu'ils aient été incendiés. C'est Emmanuel Fourdin en 1869 qui les fera entrer dans l'historiographie de l'autodafé61. 


\section{Les fêtes sous la révolution}

Les fêtes traditionnelles deviennent des festivités dangereuses, surtout dans un contexte de psychose contre-révolutionnaire $\underline{62}$. Des mesures sont prises pour atténuer les effets de la ducasse, manifestation multiséculaire par excellence. En 1794, le cortège est interdit, les géants sont brûlés, les plaisirs sont bridés.

Néanmoins, durant un an, des processions publiques pourront encore avoir lieu. On peut citer à titre d'exemples : une procession le dimanche de la Trinité (31 mai 1795) et la procession du Saint-Sacrement le jour de la Fête Dieu (4 juin 1795)63. Le charpentier Jean Jacques Hotton, entrepreneur des ouvrages de charpenterie 64 recevra encore 50 livres pour la construction des autels des processions jusqu'au 21 thermidor an III (8 août 1795)ㄷ․ Ce jour correspond à la publication à Ath de l'arrêté du 4 thermidor (22 juillet 1795)relatif à l'exercice du culte66. L'article 1 de l'arrêté est formel : Il ne sera fait provisoirement aucune procession hors de l'enceinte des églises. C'en est fini des fêtes religieuses, place aux fêtes révolutionnaires $\underline{67 .}$

De 179568 à 1800, la Convention thermidorienne adopte un mouvement destiné à transcrire dans un système festif construit, à visée pédagogique, l'expression symbolique des nouvelles valeurs révolutionnaires, et la prise en compte par la Révolution de sa propre histoire 69 . Les manifestations républicaines reprennent les vieux principes d'éducation des masses et de la propagande idéologique enrobés dans de grandes mises en scène.

En rupture avec le calendrier liturgique, le Directoire propose des célébrations anniversaires $\underline{70}$, des fêtes morales 71 , des fêtes décadaires $\underline{72}$ ou encore des célébrations occasionnelles en commémoration d'un héros de la République ou d'un événement militaire. Ces fêtes sont étroitement contrôlées et stéréotypées, en vue notamment de contenir en quelque sorte l'agitation populaire 73 . Le scénario est classique 74. La veille, cloches, carillon ou salves d'artillerie annoncent la fête française. Le jour même, les cloches appellent à la formation du cortège constitué d'un corps de musique, des élèves des écoles, des pensionnaires des hospices, des autorités civiles et militaires et de tous les citoyens qui le souhaitent. Précédé d'un détachement de la gendarmerie ou de la garnison, le cortège se rend au Temple de la Loi ou au pied de l'arbre de la Liberté pour une cérémonie riche en discours, hymnes et chants. L’après-midi, il peut y avoir une évolution militaire sur la place d'armes et le soir l'un ou l'autre bal.

D'aucuns $\underline{75}$ s'accordent à décrire les fêtes du Directoire en terme d'échec; un manque de spontanéité de la population ressort de la lecture des procès-verbaux. À partir de l'an VII, le Temple de la Loi ne fait plus recette; un retour en force de la dimension profane et même religieuse de la fête va progressivement se manifester. Ainsi, le Conseil des Anciens et le Conseil des Cinqcents, sur proposition des Consuls, promulguent la loi relative aux fêtes nationales le 3 nivôse VIII (24 décembre 1799)⒍ Il n'est plus désormais célébré que deux fêtes qui rappellent les époques mémorables de la révolution, à savoir l'anniversaire du 14 juillet 1789, jour de la conquête de la liberté $\underline{77}$, et le $1^{\mathrm{er}}$ vendémiaire, jour anniversaire de la fondation de la République. Toutes les autres sont supprimées.

Le 15 messidor IX (4 juillet 1801), le préfet Garnier invite les localités du département de Jemappes à célébrer le 14 juillet par des cérémonies simples et respectables où les fêtes et les jeux puissent respirer le sentiment de la félicité publique78. À Ath, pour l'occasion, divers éléments réapparaissent dans le cortège $\underline{79}$ : la compagnie des canonniers bourgeois (ex-confrérie de Sainte 
Marguerite) à titre de service d'honneur et un char triomphal à l'antique qui était déjà sorti pour la fête de la proclamation des Consuls du 10 germinal IX (31 mars 1801). Les archers des sociétés de tir se joindront à eux le $1^{\mathrm{er}}$ vendémiaire pour la fête de la fondation de la République.

Après la nomination à vie du Premier Consul (14 thermidor X - 2 août 1802) et le couronnement de l'empereur (11 frimaire XIII - 2 décembre 1804), l’État français inaugure un culte à Bonaparte reléguant les fêtes nationales au rancard. Le décret impérial du 19 février 1806 institue le 15 août comme fête de saint Napoléon et du rétablissement du culte catholique en France 80 et le $1^{\text {er }}$ décembre comme jour anniversaire du couronnement et de la bataille d'Austerlitz $\underline{81}$. Le 28 juillet, le préfet recommande aux maires de faire célébrer dignement le 15 août dans son département de Jemappes82. Monseigneur François Joseph Hirn, évêque du nouveau diocèse de Tournai, écrit une lettre pastorale le 14 mai 1806 introduisant auprès de ses fidèles le décret du 19 février83. Il ordonne que soient désormais célébrés le 15 août l'office de l'Assomption et la commémoration de saint Napoléon, martyr. Le même jour, dans chaque commune, il y aura une procession hors de l'église, en l'absence du Saint-Sacrement et précédée d'un discours de circonstance. Les autorités militaires, civiles et judiciaires seront invitées à la solennité.

\section{6 : le retour des géants}

La loi du 19 février 1806 célèbre le rétablissement du culte catholique. C'était la suite logique du Concordat conclu par Napoléon et le Pape Pie VII le 26 messidor IX (15 juillet 1801) et transcrit dans la loi du 18 germinal X (8 avril 1802), complété d'articles organiques. Trois mois plus tard, François Joseph Hirn avait été nommé à la tête du diocèse de Tournais4 ; le décret du 23 vendémiaire XII (16 octobre 1803) allait définir la nouvelle organisation de son diocèse. Avec le Concordat, les expressions du culte catholique vont pouvoir reprendre place dans la rue, au même rang que d’autres cérémonies publiques $\underline{85}$.

L'historiographie traditionnelle en matière de ducasse d'Ath tend à assimiler le cortège à la sortie des géants. Tel n'a pas toujours été le cas. Ainsi, après la Guerre de la Ligue d'Augsbourg, la procession se déroule correctement en 1698 et 1699, mais il faut attendre 1700 pour voir réapparaitre le géant Samson complètement remis à neuf6․ La situation se représente à l'aube du $\mathrm{XIX}^{\mathrm{e}}$ siècle. La première mention des festivités de la kermesse post révolutionnaire est datée du 8 fructidor XII (26 août 1804). Le règlement du jeu de l'arc qui doit avoir lieu ce jour de la kermesse de cette ville d'Ath stipule en son article 6 que les tireurs se rendront ensemble avec les sociétés à la procession qui se fera après la messe87.

La ducasse figurative «traditionnelle » s'étoffe en 1806. L'ordre de la marche de la procession de la dédicace du 24 août 180688 recense quelque 47 groupes. La marche s'ouvre par les pensionnaires des hospices des orphelins et des vieillards et par les élèves des écoles primaires. Le défilé des corps de métiers placés sous le patronage d'un saint est entrecoupé de cinq cavalcades composées de représentants de trois à quatre villages des environs. La seconde partie de la procession débute par les étudiants. Après les bergers et les représentations de la Vierge, le clergé et une musique introduisent les autorités administratives et judiciaires. La gendarmerie ferme la marche. Deux chars figurent dans le cortège.

L'ordre de marche de la procession indique la présence de quatre géants. L'Aigle est situé entre les charpentiers de saint Joseph et la $2^{\mathrm{e}}$ cavalcade, Samson et la compagnie des canonniers entre les 
menuisiers de saint Nicolas et la $4^{\mathrm{e}}$ cavalcade. Monsieur et Madame Goliath sont installés entre les étudiants et les bergers. En 1807, la figure dite Tyran viendra s'intercaler entre les tanneurs de saint Barthélemy et l'éventuelle $5^{\mathrm{e}}$ cavalcade ou les étudiants. Un article de presse anonyme publié en $1852 \underline{89}$ attribue la réalisation des têtes des géants à l'un des frères Florent, Gabriel ou Éloy Emmanuel90. Il n'a pas été possible jusqu'ici d'identifier l'un ou l'autre comme le concepteur de ces œuvres d'art, ou de clarifier les modalités d'une éventuelle collaboration des deux frères. Auteur de la statue de saint Pierre de la chaire de vérité de l'église de Leuze, Gabriel Florent est orfèvre et s'improvisera architecte en dressant les plans de l'église Saint-Julien après l'incendie de 1817. Aussi parfois qualifié de peintre, Éloy Florent est maître sculpteur et menuisier. M. Florent sera rétribué par la caisse communale en 1808 pour compte de ce qui m’est dû.

La géante Victoire - conçue en 1793 pour célébrer les revers de l'armée française et dont l'existence avait été éphémère -, ressuscitera d'une manière inattendue en 1860 lorsque la population l'identifiera au Génie féminin de la Ville créé cette année-làg1. En 1807, les boulangers tenteront de reconstruire leur cygne mais échoueront faute d'un financement suffisant $\underline{2}$.

Le programme du cortège de 1806 se veut un calque de l'ordonnancement de la procession d'Ancien Régime avec quelques nuances, comme la présence de cavalcades. L'administration municipale tend à mettre la main sur les géants en un temps qui a connu l'abolition des corporations. Hormis peut-être Samson, les postures d'osier ne sont plus attachées à un groupe et leurs têtes de tilleul appartiennent au patrimoine communal. L'ordre de marche est arrêté par le maire. Le premier magistrat de la ville nomme également des maîtres de cérémonie chargés de la police du cortège et convoque les groupes $\underline{93}$. Comme à l'été 1752, tout est conçu afin de faire le tout à l'accoutumée et de requérir les corps de stile, confréries et sermens de faire marcher à la procession leurs postures et ornemens servans à la procession $\underline{94}$.

De 1806 à 1808, le clergé amplifie la pompe religieuse $\underline{95}$. Comme par le passé, en 1807, il se rend à l'hôtel de ville pour inviter l'autorité communaleg6. En deux ans, le nombre de châsses et de gonfanons est doublé. Il n'empêche que la commune intervient dans le recrutement des porteurs et fait restaurer des statues.

\section{9 : une circulaire à / en contretemps}

Le calme instauré par le régime napoléonien et la restauration du culte sont troublés par une circulaire hollandaise datée du 29 mai 181997. Ayant constaté des abus qui résultent des processions qui ont lieu hors de l'enceinte des églises, le roi Guillaume charge son Directeur général aux Affaires du Culte catholique d'informer les évêques et gouverneurs de province que désormais il n'y aura plus, dans chaque paroisse, que deux processions $\underline{98}$ : l'une le dimanche qui suit la Fête-Dieu et l'autre au choix de l'évêque. Selon Emmanuel Fourding9, le choix de Mgr Hirn 100 détermine que la deuxième procession soit fixée au $3^{\mathrm{e}}$ dimanche de juillet, pour la fête du Saint-Sang de Miracle101. Il semble qu'elle ne soit sortie qu'une fois et que le doyen Defrenne ait sollicité son transfert au 15 août.

Il est clair que cette circulaire de mai 1819 coïncide quasi trait pour trait au décret de mai 1786. Si en 1786, le décret a suscité protestation et requête d'interprétation, il n'en est rien de la circulaire de 1819. À ce jour, les archives communales ne livrent aucune information quant à la mise en application de la circulaire102. Les seules relations échangées avec les Affaires du Culte catholique 
portent sur la reconstruction de l'église Saint-Julien après l'incendie du 10 avril 1817103, dont la première pierre est posée le 9 août 1819104. Néanmoins, l'information aux chefs diocésains ne permet aucun vêtement extraordinaire, aucune bigarrure, ni aucune représentation inconvenante, qui causent souvent des désordres et du scandale et qui toujours détruisent plus ou moins le respect que le peuple doit porter à ces actes religieux. Le cortège d'Ath entrerait-il encore dans le champ d'application de la circulaire ?

L'impact de la circulaire de 1819 est minime et ne cause pas d'émoi particulier de la part du Magistrat athois. Depuis 1806, les programmes notent une fête contenant des éléments religieux, mais organisée par les édiles communaux. Les géants sont propriété de la ville et portés par des hommes rémunérés par elle105. Dès la fin du XVIII ${ }^{\mathrm{e}}$ siècle, les semainiers de l'église Saint-Julien annoncent la procession de la ville106. Alors qu'il y a deux paroisses dans la cité, cette indication témoigne d'une part du caractère identitaire urbain pris par le cortège et d'autre part de son détachement vis-à-vis de la paroisse où le patron est fêté par une messe le jour de son anniversaire et par l'exposition de ses reliques $\underline{107}$.

Bien que d'une portée minime, la circulaire de 1819 n'en est pas moins symbolique. Psychologiquement, l'esprit de la révolution française a fait son œuvre. Bien qu'elle eût été organisée officiellement par la Ville dès le milieu du XVIII ${ }^{\mathrm{e}}$ siècle, la Ducasse conserve son caractère religieux au-delà de 1786. Le clergé tente de réinvestir son cortège dès 1804-1806. Mais il est patent de constater qu'en 1819, la fête mise sur pied par le pouvoir civil est devenue une fête communale, dont les éléments religieux ne sont plus que résiduels. En 1822 par exemple, la Commune invite les sociétés de tir à l'arc et de jeu de balle à venir encourir au gain des prix qui se donneront à l'occasion de la Fête communale qui aura lieu le dimanche 25 août présent mois108.Les affiches des programmes de la première moitié du XIX ${ }^{\mathrm{e}}$ siècle jusqu'à la seconde guerre mondiale portent la mention fêtes communales 109 .

\section{Conclusion}

La période de 1786 à 1819 est probablement l'époque où il a été le plus légiféré sur la ducasse d'Ath. Les conceptions religieuses de Joseph II, l'acharnement des patriotes jacobins, la politique thermidorienne, la restauration napoléonienne du culte et l'influence de l'administration calviniste des Pays-Bas ont asséné des coups de boutoir à une fête séculaire.

L'année 1819 peut être considérée comme une balise qui consacre le terme d'un processus lent et progressif d'une sécularisation entamée dès la seconde moitié du XVII ${ }^{\mathrm{e}}$ siècle, probablement comme un produit de la Contre-Réforme110. D'une fête d'origine et à caractère religieux, la Ducasse se mue en une fête communale. Il faudra attendre la réforme du cortège en 1850 pour que l'aspect religieux soit réduit à sa plus simple expression : à savoir les deux géants vétérotestamentaires Goliath et Samson, le jeu-combat du samedi qui voit s'affronter le berger David et Goliath le Philistin, et quelques autres éléments mineurs et moins spectaculaires (la Fuite en Égypte, saint Martin à cheval, saint Michel et le diable).

Par-delà l'arsenal normatif, les habitants d'Ath ont su conserver et maintenir intact, durant une période trouble de leur histoire, l'élément culturel devenu identitaire pour leur cité. La transmission de leur patrimoine immatériel a surmonté les guerres et les révolutions, comme peut le prouver cet extrait d'un courrier adressé par le maire au sous-intendant de l'Arrondissement de Tournai le 3 
décembre 1814 : Nous n'avons en cette ville qu'une fête communale, dite la dédicace ou carmesse, qui n'a jamais été supprimée, pas même par la révolution française. Elle a lieu le quatrième dimanche d'août. Il se fait le jour de cette fête une procession à laquelle se porte la chasse de saint Julien, patron de la paroisse, qui fait le tour de la ville à l'extérieur. Des figures gigantesques marchent à cette procession et cette fête date de 1415, époque de la cessation du grand schisme d'occident. Elle n'a rien que d'innocent et amusant111.

\section{Notes}

1 Le patrimoine culturel immatériel correspond aux «pratiques, représentations, expressions, connaissances et savoir-faire - ainsi que les instruments, objets, artefacts et espaces culturels qui leur sont associés - que les communautés, les groupes et, le cas échéant, les individus reconnaissent comme faisant partie de leur patrimoine culturel. Ce patrimoine immatériel, transmis de génération en génération, est recréé en permanence par les communautés et groupes en fonction de leur milieu, de leur interaction avec la nature et de leur histoire, et leur procure un sentiment d'identité et de continuité, contribuant ainsi à promouvoir le respect de la diversité culturelle et la créativité humaine. » ; Convention pour la sauvegarde du patrimoine culturel immatériel, Paris le 17 octobre 2003, art. 2 : définitions, publiée dans Patrimoine culturel immatériel, Paris, Unesco, s.d. (Documents).

2 Chefs-d'œuvre du patrimoine oral et immatériel de l'humanité. Proclamations 2001, 2003 et 2005, s. dir. D. SMEETS, Unesco, 2006.

$\underline{3}$ L'habitude a été prise dès les origines de faire sortir la procession soit le dimanche coïncidant au 28 août (fête de Saint-Julien de Brioude) soit le dimanche précédant cette date, autrement dit, en tous les cas, le dimanche avant le 29 août (fête de la Décollation de Saint-Jean-Baptiste) ou, pour reprendre la terminologie en usage actuellement, le $4^{\mathrm{e}}$ dimanche d'août.

4 Pour un aperçu synthétique de l'histoire de la ducasse d'Ath, consulter : E. FouRDIN, La procession et la foire communale d'Ath, dans Annales du Cercle archéologique de Mons, t. IX, 1869, p. 1-69 ; R. MEURANT, Le cortège d'Ath du XVe au XIXe siècle, dans La Ducace d'Ath. Etudes et documents, Bruxelles, Commission royale belge de Folklore, 1981, p. 23-49 (Folklore et art populaire de Wallonie, VII) et J.-P. DuCAStelle et L. Dubuisson, La Ducasse d'Ath. Passé \& présent, Ath, Maison des Géants, 2014.

$\underline{5}$ Des exemples en sont donnés par C. CANnUYER et a., Goliath opprimé, Goliath libéré. $50^{e}$ anniversaire de la Libération d'Ath et de la Ducasse, Ath, 1994 ; J.-P. DENIS, La procession de la dédicace au XVIIIe siècle au travers d'archives peu ou pas exploitées, dans Bulletin du Cercle royal d'histoire et d'archéologie d'Ath et de la région (B.C.R.H.A.A.), $\mathrm{n}^{\circ}$ 160, juillet 1994, p. 449-471 et A. Dupont, Samson opprimé, Samson libéré. Étude sur le Samson d'Ancien régime, dans B.C.R.H.A.A., $\mathrm{n}^{\circ}$ 280, juillet 2014, p. 633-657.

$\underline{6}$ G.J. DE Boussu, Histoire de la ville d'Ath, Mons, 1750, p. 146-147.

7 J.-P. DeNIS, La procession de la dédicace au XVIIIe siècle, op. cit., p. 449-471 ; A. DUPONT, Samson opprimé, op. cit., p. 638-643.

8 Le programme avait été encarté dans le « Programme de l'ordre et de la marche de la procession de la dédicace de la Ville d'Ath le 23 août 1807 »; Archives de la Ville d'Ath (A.V.A.), 
Fêtes communales, F.C. 2.1. ; R. MEURANT, L'ordonnance de la procession d'Ath au XVIIIe siècle. Un document inédit, dans L'Écho de la Dendre, 27 août 1966 et reproduit dans La Ducace d'Ath, op. cit., p. 51-54. La plupart des textes antérieurs à 1794 importants sur la ducasse ont été dépouillés par René Meurant et édités dans La Ducace d'Ath, op. cit.

9 A. Hoverlant de Bauwelaere, Essai chronologique pour servir à l'histoire de Tournai, t. 68, 1820, p. 25-28. Pour une analyse de cette description, lire : J.-P. Ducastelle, La Ducasse d'Ath vue par un écrivain tournaisien (XVIIIe - XIXe siècles), dans B.C.R.H.A.A., $\mathrm{n}^{\circ}$ 154, juillet 1993, p. 329-333.

10 Recueil des ordonnances des Pays-Bas autrichiens (R.O.P.B.A.), $3^{\mathrm{e}}$ série : 1700-1794, t. XII, sous la dir. de P. VerhaEghen, Bruxelles, 1910, p. 470. A.V.A., Généralités, Édits et décrets imprimés émanant du gouvernement, G 213, pièce 154 .

11 Archives générales du Royaume (A.G.R.), Conseil Privé, cartons de la période autrichienne, $\mathrm{n}^{\circ} 704 \mathrm{~A}$.

12 A.V.A., Imprimés du régime autrichien, à la date.

13 A.V.A., Généralités, Délibérations de la Chambre échevinale (1784-1794), G 11, fº 38 rº.

14 A.V.A., Généralités, Lettres expédiées par l'autorité communale (1779-1794), G 218, f 63 v $64 \mathrm{r}^{\circ}$.

15 E. Roland, Philippe-Jacques Defrenne, ancien curé et doyen d'Ath. Son opposition au Concordat et à Monseigneur Hirn. Son exil (1801-1814), dans Annales du C.R.H.A.A., t. XXXV, 1951, p. 29-63 ; E. et M. Roland, Philippe Jacques Defrenne (II), dans Annales du C.R.H.A.A., t. XLIX, 1983, p. 225-235.

16 À l'époque Ath relève du diocèse de Cambrai, dont le siège est établi en territoire français.

17 E. et M. Roland, op.cit.. Au Conseil privé, les dispositions portant sur l'abolition des jubilés et autres démonstrations dans ces pays sont confiées à Monsieur de Limpens (A.G.R., Conseil Privé, registres, $\mathrm{n}^{\circ} 55, \mathrm{f}^{\circ} 172 \mathrm{v}^{\circ}$ en date du 24 avril 1786) qui s'intéresse principalement aux cérémonies du Saint Sang de Bruges (A.G.R., Conseil Privé, registres, $n^{\circ} 208, \mathrm{f}^{\circ} 156 \mathrm{r}^{\circ}-158$ vº protocole en date du 29 avril 1786). Cette question sera probablement à l'origine du décret défendant de célébrer les jubilés en mai 1786 ; A.G.R., Conseil Privé, registres, n 620, dossier 120.

18 Datée du 25 avril, elle est en copie dans A.V.A., Généralités, Livre des décrets, lois et ordonnances (1776-1786), G 15, pièce 28, fo $168 \mathrm{r}^{\circ}-\mathrm{v}^{\circ}$.

19 Subtilement, le courrier n'évoque que la procession et non les pratiques traditionnelles qui entourent l'événement. Mais il abolit toutes les démonstrations d'usage la veille de la kermesse.

$\underline{20}$ R.O.P.B.A., op.cit., t. XII, p. 470. A.V.A., Généralités, G 213, pièce 160 et Imprimés du régime autrichien, à la date.

21 A.G.R., Conseil Privé, cartons de la période autrichienne, nº $704 \mathrm{~A}$.

22 Philippe-Jacques Defrenne obtient la cure d'Ath en novembre 1778. Selon Jean Plumet, treize ans durant, il gouverna sa paroisse avec intelligence et fermeté. Il y a peu de chose à dire sur 
cette première période de son ministère à Ath. Il vécut sans doute en parfaite intelligence avec les autorités civiles de la ville; J. PLUMET, La vie mouvementée d'un curé d'Ath sous la Révolution française. L'abbé Philippe-Jacques Defrenne, doyen de Chièvres et vicaire général de Cambrai (1739-1819), dans Annales du Cercle royal d'archéologie d'Ath, t. XXXI, 1946, p. 1-16, en particulier p. 2.

23 Les différends entre les nouveaux échevins et le doyen auraient commencé en juillet 1784 à propos de la Maison des Orphelins de la Ville.

24 A.G.R., Conseil Privé, registres, $\mathrm{n}^{\circ} 209$, fo $171 \mathrm{r}^{\circ}-\mathrm{v}^{\circ}$ : protocole en date du 31 juillet 1786.

25 A.G.R., Conseil Privé, cartons de la période autrichienne, nº 704 A.

26 A.G.R., Conseil Privié, registres, n 620, dossiers n 120-121.

27 Par jubilé, le projet du décret entend une fête qui se célèbre en commémoration de quelque événement particulier.

28 Cfr note 17 : A.G.R., Conseil Privé, registres, $n^{\circ} 208, f^{\circ} 156 r^{\circ}-158 v^{\circ}$, protocole en date du 29 avril 1786.

29 A.V.A., Généralités, Correspondance émanant du gouvernement (1780-1786), G 224, pièce $\mathrm{n}^{\circ} 30$ et Livre des décrets, lois et ordonnances (1776-1786), G 15, fo $169 \mathrm{v}^{\circ}$.

30 A.V.A., Généralités, Délibérations de la Chambre échevinale (1784-1794), G 11, f 41 ro.

31 A.V.A., Comptabilité, Co 164. Les objets de la chapelle sont qualifiés de bois et vieux bois ; il s'y trouve une chapelle en bois, une statue de saint en bois et une image de saint Nicolas.

32 À titre d'exemples, A.V.A., Culte, Paroisse St-Julien, comptes de la mambournie : en 1788-1789 (SJ 886) fo $223 \mathrm{r}^{\circ}-225 \mathrm{r}^{\circ}$, en 1783-1785 (CSJ 60) fo $182 \mathrm{r}^{\circ}-\mathrm{v}^{\circ}$ et en 1780-1782 (SJ 885) fo $170 \mathrm{v}^{\circ}$ $171 \mathrm{r}^{\circ}$.

33 A.V.A., Comptabilité, Massarderie, compte 1786, $2^{\mathrm{e}}$ entremise, liste des ouvrages qu'on n'a pu prévoir auxquels il a fallu travailler d'abord, $\mathrm{f}^{\circ} 3 \mathrm{r}^{\circ}$.

34 Depuis le nouveau règlement du 9 février 1769 sur la comptabilité communale d'Ath (Règlement pour la ville d'Ath du 9 février 1769, Bruxelles, impr. De Grieck, 1772 et R.O.P.B.A., op.cit., $3^{\mathrm{e}}$ série, t. IX, s. dir. J. DeLECOURT, Bruxelles, 1897, p. 482-492) et en particulier son article 7, les ouvrages publics sont affermés. Pour la période du $1^{\mathrm{er}}$ septembre 1784 au 31 août 1787, les ouvrages de charpenterie ont été octroyés au maître charpentier Jacques Pierre Routier (A.V.A., Généralités, registres des criées, G 114, f $146 \mathrm{r}^{\circ}-155 \mathrm{v}^{\circ}$ ). L'article 5 de son contrat prévoit que le charpentier est en charge du char de la ville pour la procession de la kermesse, des deux géants, des deux chevaux postiches d'osier avec les habillements du cavalier et des autels ou reposoirs qui s'érigent sur la place lors des processions et des théâtres, ainsi que le pont qui s'érige sur la rivière pour faire passer la procession; ces ouvrages sont repris sous les $n^{\circ} 89$ à 92 de la liste des objets d'ouvrages publics à charge de la ville en conformité de l'article 7 du règlement de 1769 (A.V.A., Travaux Publics, T 9 à T 11).

$\underline{35}$ Aucune somme n'est portée en recette comme produit de la vente et aucun remplois de 
matériaux n'est signalé dans les archives communales. Leurs dépouillements opérés tant par moi, que par les historiens et folkloristes Emmanuel Fourdin, Christian Cannuyer, Jean-Pierre Ducastelle, Jean-Pierre Denis et René Meurant, n'ont pu à ce jour éclairer sur la suite à donner à cette mention de la comptabilité.

$\underline{36}$ A.V.A., Culte, Paroisse Saint-Julien, SJ 40 (semainier 1789-1794).

37 La mercuriale a été éditée par F. LEURIDANT : Le Tribunal de Première instance d'Ath en 1787, dans Annales du Cercle d'histoire d'Ath, t. VI, 1920, p. 98-99 et commentée par C. CANNUYER, Goliath d'autrefois. Études sur le géant athois Goliath d'avant la restauration de 1806, Ath, Centre de Lecture Publique, 1991, p. 30-31. Elle avait pour objet principal non la question des processions, mais celle de la réorganisation des tribunaux voulue par Joseph II : les Montois reprochaient aux Athois de l'avoir approuvée parce que leur « petite bicoque de ville » avait été dotée d'un tribunal de première instance.

$\underline{38}$ La phrase peut porter à réflexion sur deux points. Le premier est d'abord l'attachement des Athois à leur géant Goliath. Le second note que le géant gémit, il n'est donc point mort et pourrait se lamenter dans quelques sombres recoins d'un lieu de stockage.

$\underline{39}$ A.G.R., Conseil Privé, cartons de la période autrichienne, nº 704 A.

40 Lire notamment pour la situation athoise : C. CANNUYER, Echos d'une ville hainuyère aux premiers émois de la «Révolution brabançonne » : Ath 1781-1789, dans Autour de la ville en Hainaut, Ath, 1986, p. 489-517 (Etudes et documents du C.R.H.A.A., t. VII).

41 R.O.P.B.A., $3^{\mathrm{e}}$ série, t. XIII, s. dir. P. VERHAEgen, Bruxelles, 1914, p. 570-571. A.V.A., Imprimés du régime autrichien, boîte 3 (1786-1791), n 16. F. RoussEAU, Légendes et coutumes du Pays de Namur, Bruxelles, 1920, p. 61.

$\underline{42}$ Les artisans mis à contribution sont bien connus sur la place d'Ath. Ils travaillent déjà régulièrement sur les géants Goliath et Samson. J.-P. DUCASTELLE, Savoir-faire et tradition. Les artistes et les artisans athois au service des géants $\left(18^{e}-20^{e}\right.$ s.), dans B.C.R.H.A.A., $\mathrm{n}^{\circ} 214,2003$, p. 106-138 et A. Dupont, Samson opprimé, op. cit.

43 A.V.A., Comptabilité, Massarderie, compte de la seconde entremise de 1790 et liste des frais et débours pour la réparation des figures et A.V.A., Comptabilité, Co 94 : pièces justificatives 1790 .

44 A.V.A., Comptabilité, Co 19 : registre des ordonnances de paiement 1783-1794, fo $140 \mathrm{r}^{\circ}$, $n^{\circ} 625$.

45 C. CANnUYeR, Echos, op. cit., p. 495.

$\underline{46}$ A.V.A., Comptabilité, Massarderie : voir les comptes de la $2^{\mathrm{e}}$ entremise pour les années 1791 à 1793.

47 Sur cette période, consulter : J.-P. DENIS, Ath sous l'occupation française : la liberté muselée, dans Actes du colloque d'histoire à l'occasion du bicentenaire de la bataille de Tourcoing, 26 floréal II - 18 mai 1794, Mouscron, 1995, p. 211-269 (Publication extraordinaire de la Société d'histoire de Mouscron). 
48 Elle tenait une branche de laurier à la main. Sur le géant Mademoiselle Victoire, lire : J.-P. DENIS, Victoire aux deux visages : contribution à une meilleure connaissance des géants athois, dans B.C.R.H.A.A., $\mathrm{n}^{\circ}$ 166, juillet 1995, p. 79-95 ; J.-P. DuCASTELLE, Mademoiselle Victoire a 150 ans, dans B.C.R.H.A.A., $\mathrm{n}^{\circ}$ 256, juillet 2010, p. 630-636. E. WALTRE, Histoire de la ville d'Ath, Tournai, s.d. [1860], p. 97.

49 Lettre de Charles Jaubert à Louis Bouteville en date du 30 frimaire V (20 décembre 1796) ; A.G.R., Papier Bouteville, carton 37 (ancienne numérotation).

50 A.V.A., Généralités, G 12 : registre des rétroactes, f ${ }^{\circ} 136$ vº. $^{\circ}$.

51 Sur la Société populaire, voir J.-P. DENIS, Ath sous l'occupation française, op.cit., p. 229-242.

52 A.V.A., Généralités, registre aux délibérations de la municipalité 1794-1796, f 19 vº 20 rº.

53 A.V.A., Généralités, correspondance reçue, G 227, f 23 vº.

54 A.V.A., Généralités, délibération de la municipalité du 6 fructidor an II.

55 A.V.A., Généralités, correspondance émanant de l'autorité communale, G 227, fo $27 \mathrm{v}^{\circ}-29 \mathrm{r}^{\circ}$.

56 C. CANNUYER, L'autodafé des géants d'Ath en août 1794. Un nouvel élément à verser au dossier, dans B.C.R.H.A.A., nº 148, juillet 1992, p. 177-183.

57 Marie Ursule Dereux n'est pas une inconnue en matière de géants. Le 7 septembre 1795, elle épouse François Deltombe en l'église Saint-Martin. Dès 1792, à l'âge de 31 ans environ, elle procède à diverses réparations et fournitures pour l'ornement des deux figures. Son époux est maître rubanier et marchand de rubans. À ce titre, il vend de l'aunage de soie lors de la restauration des géants en 1790 .

58 A.V.A., Comptabilité, registre des ordonnances de paiement, Co 20, fo 22, mandat $n^{\circ} 227$.

59 C. CANnUYer, Goliath opprimé, op.cit. ; A. DUPOnT, Samson opprimé, op. cit. ; L. DUBUISSON, La Ducasse, par-delà les rires et les larmes, dans Vendanges de la solidarité, Ath, 27 novembre 2004, p. 59-66.

60 Cela est confirmé en 1796 dans le courrier de Jaubert à Bouteville. Comme l'a fait remarquer J.-P. Denis, la géante est une insulte permanente à la République.

61 E. FouRdin, La procession, op.cit., p. 35. J.-P. DUCASTELLE, Du nouveau sur Mademoiselle Victoire, dans B.C.R.H.A.A., $\mathrm{n}^{\circ}$ 166, juillet 1995, p. 77.

62 Cette psychose est accrue du fait des tensions sociales générées par des problèmes de subsistance. J.-P. DENIS, Ath à l'ombre de l'arbre de la Liberté : agitation populaire et problèmes de subsistance de la fin de l'an II à l'an V de la République (1794-1795), dans Annales du C.R.H.A.A., t. LIV, 1995, p. 311-356.

63 A.V.A., Culte, église Saint-Julien, SJ 41 (semainier, 1794-1805).

64 Jean Jacques Hotton, maître charpentier, a obtenu le marché des ouvrages des charpenteries par criée du 26 juillet 1793 pour une période de trois ans jusqu'au 31 octobre 1796. A.V.A., 
Généralités, registre des criées, G 116, fo $21 \mathrm{r}^{\circ}-32 \mathrm{v}^{\circ}$.

65 A.V.A., Travaux publics, $\mathrm{T} 11, \mathrm{f}^{\circ} 102 \mathrm{v}^{\circ}$.

66 A.V.A., Généralités, Livres des décrets, lois et ordonnances, G 17, fo 133 v. Pasinomie ou collection complète des lois, décrets, arrêtés et règlemens généraux qui peuvent être invoqués en Belgique, $1^{\text {ère }}$ série, t. VI, s. dir. J.-B. Duvergier et I. Plaisant, Bruxelles, 1834.

67 La religion aura encore à souffrir de l'ire révolutionnaire : les portes des églises Saint-Martin et Saint-Julien seront scellées le 19 frimaire an VI (9 décembre 1797) [A.V.A., Culte, église SaintJulien, CSJ 594] et la croix de la flèche de l'église Saint-Julien sera enlevée afin que ce signe de fanatisme ne frappe plus les yeux des philosophes républicains le 13 pluviose an VI ( $1{ }^{\mathrm{er}}$ février 1798) [A.V.A., Culte, église Saint-Julien, CSJ 171].

68 Le début de ce mouvement est contemporain de l'annexion des anciens Pays-Bas autrichiens à la France le 9 vendémiaire IV ( ${ }^{\mathrm{er}}$ octobre 1795).

69 M. Vovelle, Fêtes révolutionnaires, dans Dictionnaire historique de la Révolution française, sous la dir. d'A. Soboul, P.U.F., 1989, p. 450-451. Voir aussi : J.-P. DENIS, Ath sous l'occupation française, op.cit., p. 242-245 et R. MEURANT, Fêtes publiques à Ath sous le régime français, dans La Ducace d'Ath, op.cit., p. 69-86.

70 Par exemple : le 21 janvier, anniversaire de l'exécution de Louis XVI ; le 2 février, anniversaire de la juste punition du dernier roi des Français ; le 20 mars, fête de la souveraineté du Peuple ; le 14 juillet, jour de la conquête de la Liberté ; le 4 septembre, commémoration du coup d'Etat républicain de l'an $\mathrm{V}$; le 22 septembre, fête de la fondation de la République.

71 Comme la Jeunesse, les vieillards, l'agriculture ou la reconnaissance.

72 Ces fêtes sont destinées à remplacer le dimanche.

73 Entre autres, consulter pour Ath : J.-P. DENIES, Ath à l'ombre de l'arbre de la Liberté, op. cit.

74 R. MeUrant, Fêtes publiques, op.cit., p. 71. A.V.A., Imprimés du régime français, IRF 446 : affiche de la marche à suivre pour les administrations municipales du canton de Mons lors de l'anniversaire de la fondation de la République le $1^{\mathrm{er}}$ vendémiaire $\mathrm{V}$.

75 M. Vovelle, op. cit. ; R. Meurant, Fêtes publiques, op. cit. et J.-P. Denis, Ath sous l'occupation française, op.cit..

76 Bulletin des Lois de la République française, $\mathrm{n}^{\circ} 340$; Recueil des loix de la République française et des actes des autorités constituées depuis le régime constitutionnel de l'an VIII, t. I cahier $1^{\mathrm{er}}$, Bruxelles, impr. G. Huyghe, s.d., p. 198-199. La Constitution de l'an VIII du 23 frimaire (13 décembre 1799) entre en effet en vigueur ce 3 nivôse, après le coup d’État de Bonaparte le 18 brumaire (9 novembre).

77 Pour l'organisation de cette fête à Ath, voir A.V.A., Fêtes publiques, dossier F.P. 1.2

78 A.V.A., Imprimés du régime français, IRF 316. 
79 R. MEurant, Fêtes publiques, op.cit., p. 72-73.

$\underline{80}$ Fêter la Saint-Napoléon le 15 août est une façon pour l'empereur d'atténuer l'importance de la fête de l'Assomption au moment où le Concordat vient d'être signé. Sur l'organisation de cette fête à Ath, voir A.V.A., Fêtes publiques, F.P. 1.12

81 Sur l'introduction et l'organisation de cette cérémonie à Ath, voir A.V.A., Fêtes publiques, FP 1.1 et F.P. 1.3

82 Répertoire administratif du Hainaut ..., J.-B. BIVORT, Mons, 1838, p. 129.

83 Archives de l'Evêché de Tournai, Lettres pastorales, dossier B/1/2.

84 A. Milet, Mgr François-Joseph Hirn (1751-1819), premier évêque concordataire du diocèse de Tournai. Un épiscopat difficile !, Tournai - Louvain-la-Neuve, 2002 (Tournai. Art et Histoire, 16).

85 Le décret du 24 messidor XII (13 juillet 1804) en matière de cérémonies publiques règle les préséances et les honneurs civils et militaires.

86 A. Dupont, Samson opprimé, op.cit., p. 645 et 653.

87 A.V.A., Fêtes communales, dossier FC 2.1 : règlement du 5 fructidor XII.

$\underline{88}$ A.V.A., Fêtes communales, dossier FC 2.1

89 L'Impartial. Journal des intérêts moraux, matériels et politiques de la ville et de l'arrondissement d'Ath, $\mathrm{n}^{\circ} 32,2^{\mathrm{e}}$ année, dimanche 8 août 1852, p. 1-2.

90 Sur les têtes des géants, consulter : J.-P. DuCASTELLE, Les têtes des géants d'Ath, dans B.C.R.H.A.A., n 148, juillet 1992, p. 185-201 et Les têtes des géants, chefs-d'œuvre du patrimoine mobilier de la Communauté française, dans B.C.R.H.A.A., $\mathrm{n}^{\circ} 246$, juillet 2011, p. 85-88.

91 J.-P. DuCASTELle, Mademoiselle Victoire a 150 ans, op. cit., p. 635-636.

92 R. MEURAnt, Fêtes publiques, op.cit., p. 79 et C. CANNuYER, Aux jardins secrets des géants d'Ath, Ath, 1992, p. 13.

93 A.V.A., Fêtes communales, dossier FC 2.1

94 A.V.A., Généralités, Résolutions du Conseil de ville (1729-1753), G 7, f $252 \mathrm{r}^{\circ}-\mathrm{v}^{\circ}$ : résolution du 24 juillet 1752 .

95 A.V.A., Fêtes communales, F.C. 2.1 ; R. MEURANT, Fêtes publiques, op. cit., p. 75-78.

$\underline{96}$ À partir de 1809, il n'ira plus qu'au pont du Gâdre, à mi-chemin entre l'église et l'hôtel de ville.

97 Recueil des circulaires, instructions et autres actes émanés du Ministère de la Justice ou relatifs à ce département, $2^{\mathrm{e}}$ série (1814-1830), t. II (1817-1824), Bruxelles, 1850, p. 184. En réalité, il y a deux circulaires. Celle du 2 juin 1819 vient interpréter celle du 29 mai. Au cas où il existe plusieurs paroisses dans une même ville, d'une part la procession de la Fête-Dieu sera commune aux paroisses et d'autre part, il est invité à ce que la seconde procession le soit 
également. Voir aussi R. MEURANT, Les avatars du cortège de la ducace d'Ath, dans La Ducace d'Ath, op. cit., p. 88.

98 Dans le courrier aux évêques, les Rogations sont autorisées.

99 E. FOURDIN, La procession et la foire, op.cit., p. 36-37, n. 2.

100 Mgr Hirn décèdera quelques semaines plus tard en juillet 1819.

101 Le Saint-Sang de Miracle est une des quatre processions générales d'Ath sous l'Ancien Régime. Nous aurons l'occasion de revenir sur ce concept de procession générale dans un article ultérieur.

102 Le silence est total tant dans les archives de l'administration communale que dans les archives paroissiales. De plus, l'historiographie actuelle n'a connaissance de la circulaire que par la découverte de son texte dans la Pasinomie de 1850 et non par les archives communales.

103 A.V.A., Généralités, Registres de la correspondance envoyée, G 239 (1817-1819) et G 240 (1819-1823).

104 A.V.A., Généralités, Registre des délibérations du Conseil de Régence (1819-1822), f 33 v $^{\circ}$ : délibération du 9 août 1819.

105 Voir notamment les mémoires de ce qu'a livré la veuve Delhaye aux porteurs de 1817 à 1820 ; A.V.A., Fêtes communales, F.C. 2.1.

106 A.V.A., Culte, Paroisse Saint-Julien, SJ 40 (semainier, 1789-1794).

107 Quelle qu'ait été la méthode utilisée par René Meurant lors de ses dépouillements, leur édition révèle que le dernier quart du $\mathrm{XVI}^{\mathrm{e}}$ siècle et le premier du $\mathrm{XVII}^{\mathrm{e}}$ siècle ont vraisemblablement marqué une transition dans le prise en charge de l'organisation de la procession, qui est passée de la mambournie de l'église Saint-Julien à la massarderie de la ville d'Ath.

108 A.V.A., Fêtes publiques, F.P. 2.1.

109 J.-P. Ducastelle, La Ducasse d'Ath, Bruxelles, Ministère de la Culture de la Communauté française de Belgique, 1994 (La Tradition par l'image, 1).

110 Le géant Goliath passe du giron de la confrérie des arbalétriers à la Ville. Il est doté d'une épouse en 1715. En 1751, comme à l'accoutumée, le Conseil de Ville convoque les acteurs de la procession.

111 A.V.A., Généralités, Indicateur de la correspondance (1812-1815), G 235, minute n 1051. Cette assertion est corroborée par une description de la ducasse publiée dans La Feuille de Tournay du 7 septembre 1823. Il y est d'ailleurs question de scènes fort plaisantes, surtout quand tous les mannequins dansent; édition de la description dans : P. HERMAN, A Ath, il y a un siècle et demi. La ducace - le cortège, dans L’Echo de la Dendre, 21 août 1976. 
C@hiers du CRHIDI 2406-4157 Vol. 38 - 2016, 254

Url de l'article : https://popups.uliege.be:443/1370-2262/index.php?id=254 